\title{
Immersive Simulation in Instructional Design Studios
}

\author{
> Antonieta Angulo \\ Ball State University, United States \\ aangulo@bsu.edu
}
> Guillermo Vásquez de Velasco
Ball State University, United States
guillermo@bsu.edu

\begin{abstract}
The paper describes the implementation of "immersive simulation studios" at Ball State University and their impact on architectural design education. This implementation is part of our on-going research efforts aimed to expand conventionally available digital design tools by including state-of-the-art virtual reality (VR) technology in design studios. Two consecutive immersive simulation studios were held during the academic year 2013-2014; we tested teaching/learning methodologies for effectively using the VR simulation to support the students in the design of architectural spaces. The results make reference to the learning outcomes from these implementations and the level of satisfaction of students using the tool.
\end{abstract}

Reywords: Architectural Education; Design Studios; Virtual Reality; Immersive Simulation; Head-Mounted Display

\section{Introduction}

In recent years, immersive VR systems have gained popularity. This popularity may be in part attributed to their lower cost and easier user interface in the implementation of games and other applications of the entertainment industry. The widespread use of these systems have prepared the current generation of design students to skillfully learn how to use VR systems. As there is progressive adoption of immersive VR systems, the systems with high interface and environment fidelity could be seen as more suitable for spatial tasks. Studies in the field state that "environment fidelity" would reflect the quality of the sensory information provided to the user by the simulator, while "interface fidelity" would relate to one's actions used to generate this information (Waller et al. 1998). The current technology of a Head Mounted Display (HMD) is highly immersive due to these specific qualities and therefore suitable for architectural design tasks that heavily rely on the visualization of space. The CAP VR Environment deployed in the College of Architecture and Planning at Ball State University, makes use of state-of-the-art HMD technology allowing a single user to navigate three dimensional models of infinite size, while physically limited to walking around a 20' x 20' room.

The CAP VR Environment provides effective immersion based on the level of presence that the users experience as a direct consequence of:

1. Very high environment fidelity depicted through the display rate of the system, the photorealistic images, lights, shadows, and the inclusion of animated avatar, and
2. Very high interface fidelity depicted through navigational aids that support way finding, path integration, spatial search, distance perception and orientation among other navigational tasks (Lathrop \& Kaiser, 2005). Additionally, we use the hand-held wand that permits free exploration of large virtual environments (VEs), especially by flying. When navigating inside VEs of their own design, we have noticed that the students rely not only on vision but also on the memory of the drawings of the same spaces as they have created them using CAD tools. See figure 1.

We believe that the CAP VR Environment should be aimed to support the actual architectural design process, therefore aiding the process of learning how to design, rather than limiting its use as a visualization tool for finalized projects. We also believe that the CAP VR Environment can better serve its purpose as a tool for simulating the architectural spatial experiences therefore transcending the depiction of the space defining objects.

During the spring term of 2012 we conducted an initial study implementing a controlled experiment with novice architectural design students (Angulo, 2013). Through that experiment we compared projects that were produced using enhanced means (digital flat media and physical models) against projects that were implemented through VR models that were displayed in the CAP VR Environment as part of the design process. We obtained a clear indication that the design projects using the VR system were the best projects in the class. We also concluded that the CAP VR Environment may have contributed with in-time feedback to promote/ 
inform changes in the conceptual design stage, and moreover the visualization of projects using the immersive environment may have had a positive impact on re-invigorating the participatory dynamics of the studio review. We have subsequently implemented two immersive simulation studios during the 2013-2014 academic year.
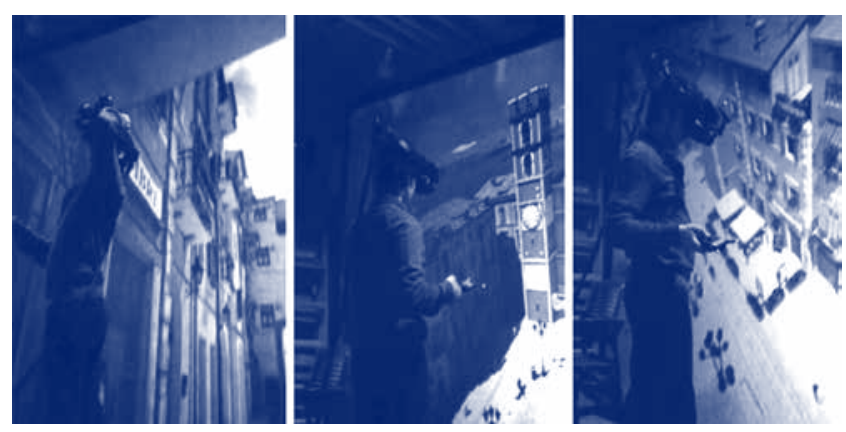

Fgure 1. Navigation within the CAP VR Environment using the HMD.

\section{Immersive Simulation Studios}

The immersive simulation design studios involved two different groups of students, the first one belonging to a $2^{\text {nd }}$ year class of the graduate program (fall 2013), and the second one belonging to a sophomore class of the undergraduate program (spring 2014). The topic of the design project was the redesign of the lobby of a small hotel in Muncie, Indiana. Due to its prominent role in greeting the guests and establishing the character of the hotel brand, the lobby was regarded as the signature space of the hotel.

The working hypotheses of this research stated that:

3. The immersive simulation in the CAP VR Environment would recreate the architectural characteristics of the space and would be effective in eliciting an appraisal of the architectural spatial experience it provides, and

4. The students will have a positive perception of their learning as they use the VR system to simulate their designs.

The project briefing, the suggested design process, the research hypothesis, and the research methodology were the same in both studios. The suggested design process undertaken by students and the research were developed in parallel supporting each other. The design/research methodology is illustrated by the diagram in figure \# 2. The figure 2 depicts two columns: one for the design process that students followed and the other for the research process. The design was performed with cyclical loops in a system that works with representation nodes and activities, after the descriptive model of design in Angulo (1995).

The design went through planning/ analysis, generation, and architectural synthesis stages. The design activities generated not

Figure 2. Research/Design Methodology.

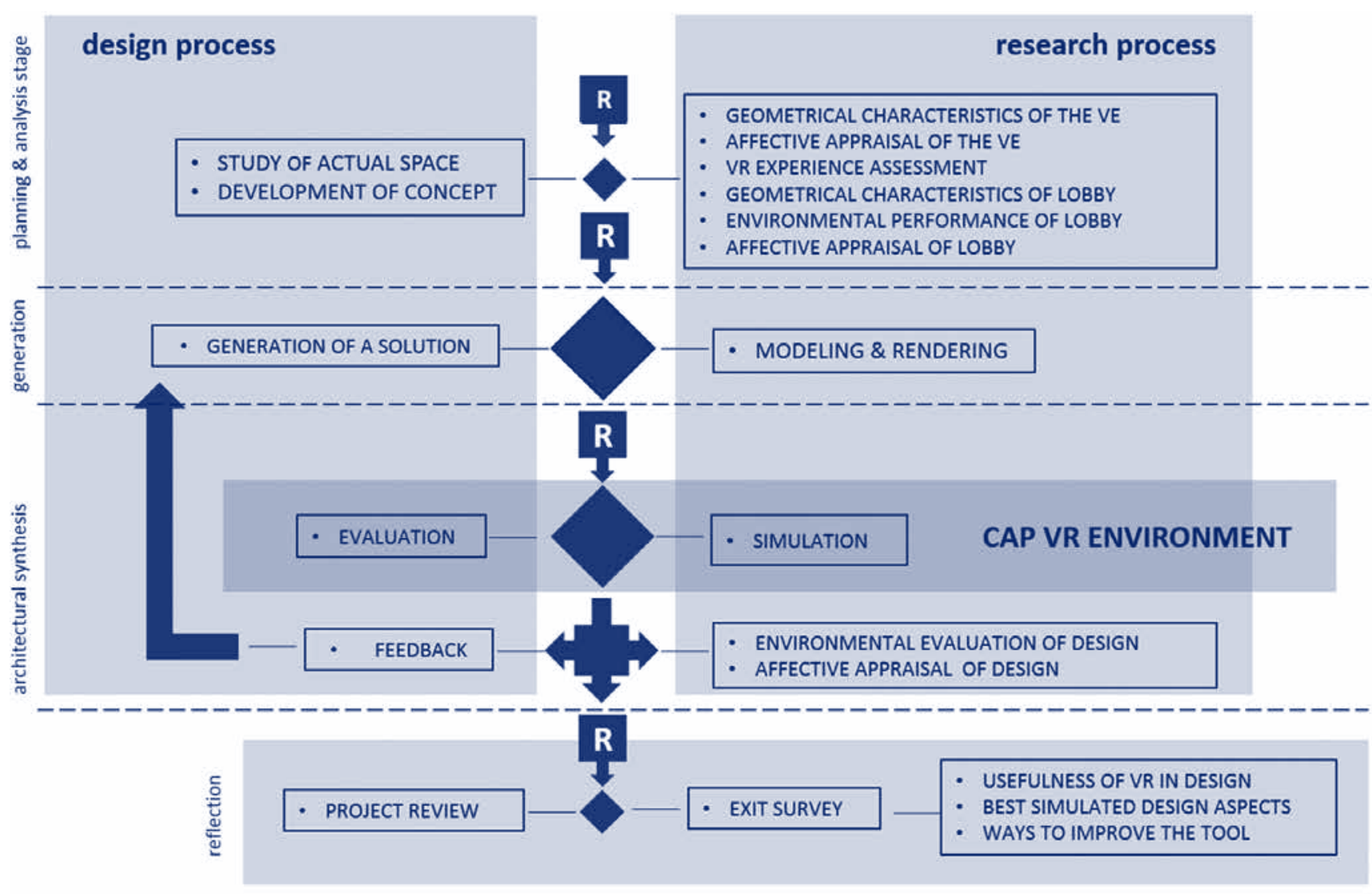

LEGEND: $\mathbf{R}$ REPRESENT $>$ OPERATE 
only more representations to be used in the following design stage but also generated research data. We used partial results from the different design stages to feed into the research process as data. Partial findings looped several times into a design/research process to generate a more comprehensive result each time.

Finally there was a reflective stage that happened after the review of the final presentation of the projects. It is important to mention that the transparency posed by the research methods affected the students' learning by exposing them to the different research metrics and making them aware of their correlation and impact in the design process.

\section{Data Collection}

During the planning and analysis stage the students ascertained not only the physical characteristics of the spaces but also had an architectural spatial experience. That was enabled using two different formats: first, the students walked through a VR model of the actual lobby using the CAP VR Environment, and second, the students visited the actual hotel. In both scenarios, the students filled out questionnaires that enquired about space dimensions, spatial orientation, first impressions of the space, and the affective appraisal of the spaces. Students provided information about the experience using the CAP VR Environment. They also produced an environmental evaluation of the actual building to be redesigned, as a method to infer criteria that became the project's working objectives.

On the development of a concept for the redesign, the students were encouraged not only to articulate how to bring solutions to discovered problems but also how to enhance the image of the signature space through the re-branding of the hotel. The creation of the alternative solutions was done using conventional 3D modeling applications. The CAD models were translated into formats used by the Vizard application (WorldViz, 2013) for real-time display in the CAP VR Environment. The models were then displayed in the immersive environment; allowing the students to evaluate the validity of the spatial geometrical relationships between objects. The images at the top in figure 3 correspond to the projects during Fall 2013, at the bottom are images of projects during Spring 2014 as visualized through the CAP VR Environment.
The ability to navigate through the simulated projects was key to experience the architectural space and attain an esthetical impression and/or recognize a symbolic meaning embedded in the solutions. The students were able to test how well the particular combinations of space defining objects could suggest meaningful appreciation correlating with the perceptual objective stated as part of the design concept. The evaluation of the alternatives using the CAP VR Environment provided timely feedback that triggered changes and improvements to the projects just before their final presentation. During the review of projects the students filled out a questionnaire making reference to the combined assessment of affective appraisal and environmental evaluation of the redesigned spaces for each one of the students' projects. Finally, the students filled out a debriefing survey regarding the perception of their learning using the CAP VR Environment; they also added recommendations for improvement of the system.

\section{Analysis \& Results}

We obtained separate results from each academic term, compared the results of both groups, and checked if there was some specific trend arising. We were aware of the differences between the two groups of students (namely undergraduate and graduate classes) mainly at the levels of design experience and expertise in the use of digital tools, but those difference did not affect the research results since the projects were reviewed within their same cadre and only the data produced was then used for comparison between the two groups. The Likert Scale format was used in all questionnaires. The average rating method was applied to establish the comparison between data from the two groups.

The most important results of the data obtained through the questionnaires during this research demonstrated that:

1. There was a difference between the physical dimensions of the model shown in the simulation of the VE and the ones estimated by the students. Almost all the students assumed that the spaces in the VE were smaller than in the model,

Figure 3. Students' projects in the CAP VR Environment.
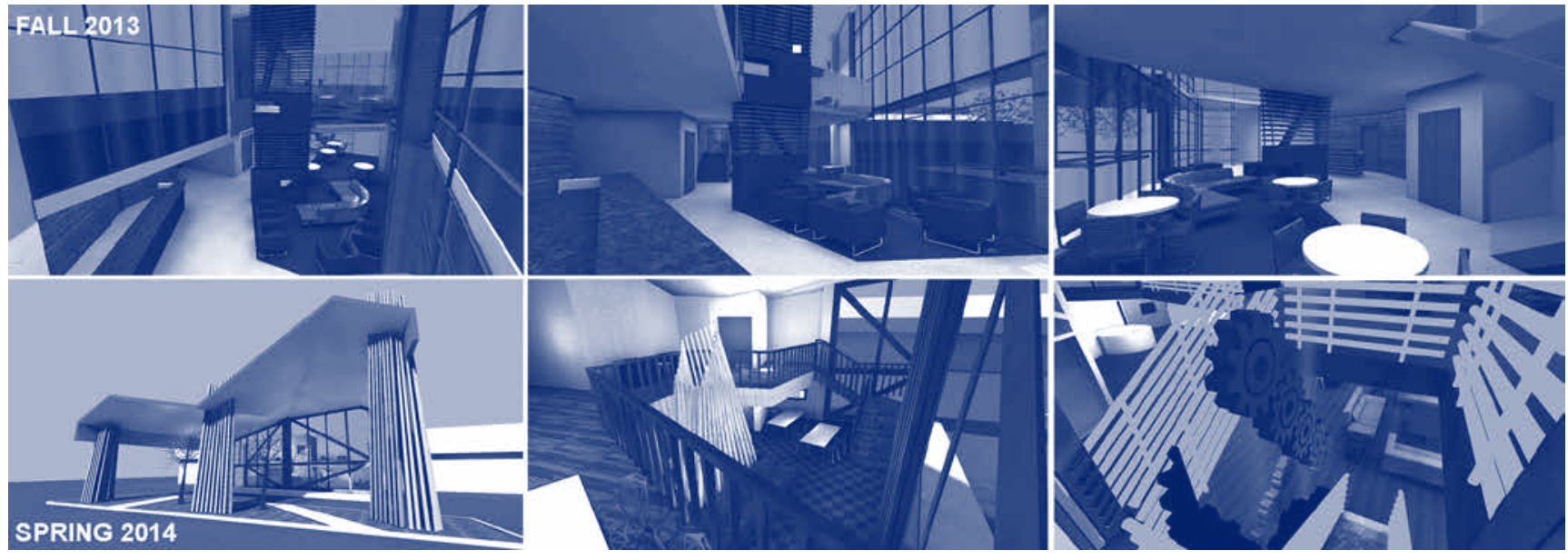


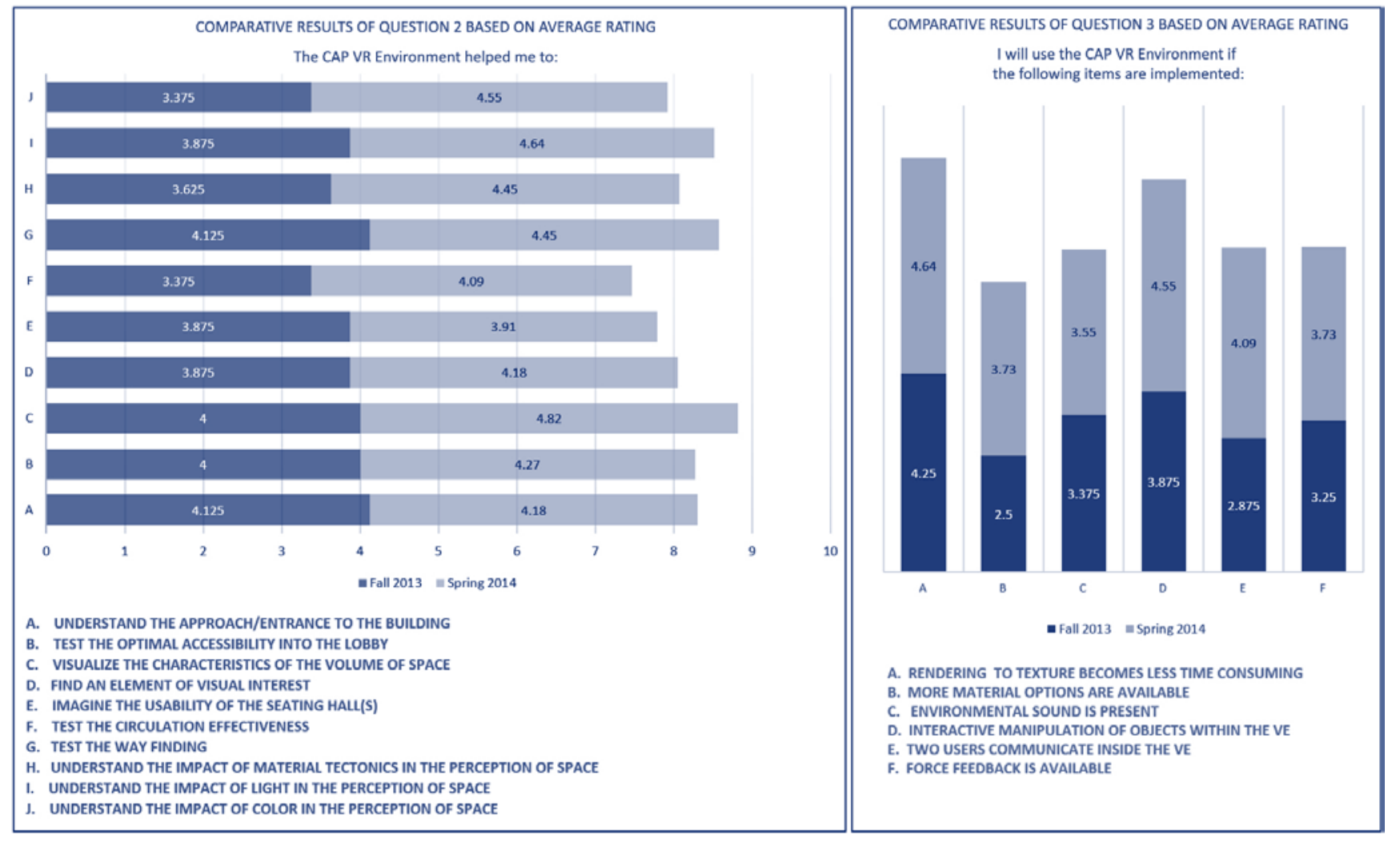

Figure 4. Results of the debriefing questionnaire.

2. Almost all the students regarded the use of the VE as a good or very good experience in agreement with the statements of the survey. .

3. The results of the "Affective Appraisal of the Space" using the VE were compared with results of the affective appraisal of reality. The average ratings in both academic terms reveal that the real experience of the space is more positive than the virtual experience due to perceptual aspects of reality missing in the scene.

4. In regard to the quality of the final projects, the results indicated that most projects were qualified as good or very good (69\%) which exceeds the benchmark of 55\% as established for this particular group and instructor in previous years.

The results of the debriefing questionnaire demonstrated that:

1. All the students in both terms "agreed" and/or "strongly agreed" with the statement "the CAP VR Environment is a useful tool to obtain feedback that no other visualization tool can provide, specifically when assessing the spatial qualities of the design" with a similar average rating in both academic semesters.

2. The design aspects that were best simulated in the CAP VR Environment related to (1) the visualization of the volume of space, (2) testing the way finding, and (3) the understanding of the impact of daylight in the space.
3. The results of the students' preference for the aspects that may need improvement in the current VR Environment revealed first that the primary concern was to further improve/facilitate/shorten the rendering process for the creation of the VEs. The second preferred aspect was to implement a capability that would allow the students to manipulate the designed objects in real-time within the VE; these modifications may include translations, rotations, and other similar transformations. Other aspects that were equally favored by the students included the addition of environmental sound and force feedback, and to establish some way of collaboration between users within the VE. The least supported aspect was the expanded availability of photorealistic material textures. See figure 4.

\section{Findings and Future Research}

The overall result of the implementation of the immersive simulation studios has provided us with enough evidence about (1) the positive benefits of using this visualization tool for the appraisal of the architectural spatial experience as seen in the quality of the finalized projects, and (2) the positive perception of students about their own learning as they use the CAP VR Environment to simulate their designs and receive constant feedback about spatial aspects that couldn't have obtained by other representational means.

We have obtained evidence towards identifying the differences between the two students' groups and the aspects that are coincidental. The two groups mainly differ in their proficiency in 
the use of three dimensional modeling and rendering tools for the production of VEs.

The graduate students may also seem more confident following a steep learning curve to generate the VR environments than the undergraduate students who struggle to achieve adequate representation quality in both the modeling and the VR production. The groups have also differed in the quality of design correlated to the amount of experience they have on designing the same subject. In both cases the differences were measured in terms of the time spent in the tasks and the quality of results obtained, being the graduates more successful. The aspect that has been coincidental for both groups of students relate to the amount of perceptual engagement they were able to maintain during the navigation of their VR environments. The perceptual engagement in the VE was effective through viewing of the spatial elements and through their ability to move around in the virtual space, physically walking or virtually navigating it with the help of the wand. The level of presence that was facilitated through this type of immersive perceptual engagement has allowed the students to assess spatial aspects that were before misunderstood or were usually left ill-defined in other projects. These aspects may include but not are limited to volume, scale, proportion, sense of presence, and expression of space. The amount of feedback that the students have received during their virtual navigation has been substantially higher if compared to the feedback usually received from conventional digital tools. The assessment derived from the VE navigation was informally absorbed within the design process but still we have not yet identified the ideal timing or establish the different design stages that would allow the students to cyclically mold and revise their designs as they navigate within gradually improved simulations.
As we look into the future we also have identified several areas for improvement that seek to make the teaching/learning about architectural space more rewarding, seek to make the system easier to use during the design process, and seek to increase the level of presence within the VE, therefore enhancing current learning outcome. Some improvements will relate to: (1) Creation of methodologies for the management of intermediate levels of visualization of the VE, (2) Procedures for real time manipulation of objects within the VE, (3) Mapping of optimal day-lighting performance with spatial effects simulated in the VE, and (4) The addition of environmental sound to the VE.

\section{References}

Angulo, A. (1995) On the conceptual feasibility of a CAAD-CAAI integrated decision support system. Delft University Press.

Angulo, Antonieta (2013) On the design of architectural spatial experiences using immersive simulation, in EAEA 11 Conference Proceedings, Envisioning Architecture: Design, Evaluation, Communication. Italy: Milan, pp 151-158.

Lathrop, W. \& Kaiser, M. 2005. Acquiring spatial knowledge while traveling simple and complex paths with immersive and nonimmersive interfaces. Presence: Teleoperators and Virtual Environments, 14(3), 249-263.

Waller, D., Hunt, E., \& Knapp, D. 1998. The transfer of spatial knowledge in virtual environments training. Presence: Teleoperators and Virtual Environments, 7(2), 129-143.

WorldViz (2013) Retrieved April, 2013 from: http://www.worldviz. com/products/vizard 